\title{
Comparison of Survival Outcomes of Lymphovascular Space Invasion- Negative and Lymphovascular Space Invasion - Positive Women with Stage IIIC Endometrioid Endometrial Cancer: A Case - Control Study
}

\author{
Zeliha FIRAT CUYLAN', Mehmet Mutlu MEYDANLI'1, Murat OZ¹, Ahmet Taner TURAN², Ozgur AKBAYIR ${ }^{3}$, \\ Murat DEDE 4 , Ali AYHAN 5
}

Ankara, Turkey

\begin{abstract}
OBJECTIVE: The purpose of this case-control study was to investigate the effect of lymphovascular space invasion on recurrence patterns and survival in patients with stage IIIC endometrioid type endometrial cancer.

STUDY DESIGN: A multicenter, retrospective, department database review was performed to identify lymphovascular space invasion-negative and lymphovascular space invasion-positive stage IIIC endometrioid endometrial cancer at five gynecological oncology centers in Turkey. Demographic, clinicopathological and survival data were collected. Cases and controls were matched in terms of age at diagnosis, grade of tumor, depth of myometrial invasion, sub-stage and adjuvant treatment.
\end{abstract}

RESULTS: Forty-one patients in the case group and 82 patients in the control group were eligible for the study. The 5-year OS rates were $94.4 \%$ in the lymphovascular space invasion-negative group and $77.2 \%$ in the LVSI-positive group ( $p=0.058$ ). Advanced age ( $\geq 58$ years) (HR 3.73; 95\% Cl: 1.19-11.69, $p=0.02$ ), grade 3 tumor (HR 2.57; 95\% Cl: 1.002-6.62, $p=0.048$ ) and cervical involvement (HR 2.62; 95\% $\mathrm{Cl}: 1.03-6.67, p=0.04$ ) appeared to be as independent predictors of decreased overall survival (OS). There was a trend towards statistical significance between the presence of lymphovascular space invasion and OS in regression analysis (HR 3.10; 95\% Cl 0.90-10.67, $p=0.05$ ). Recurrence rates were $17.0 \%(7 / 41)$ and $24.4 \%(20 / 82)$ in lymphovascular space invasion-negative and lymphovascular space invasion-positive groups, respectively $(p=0.48)$. There were 5 cases of retroperitoneal recurrences in the lymphovascular space invasion-positive group and no retroperitoneal recurrence among lymphovascular space invasion-negative women.

CONCLUSION: Lymphovascular space invasion status seems to be of borderline significance for OS in stage IIIC endometrioid endometrial cancer and impacts recurrence patterns as retroperitoneal recurrences are strongly related to positive lymphovascular space invasion status.

Keywords: Endometrioid-type endometrial cancer, Lymphovascular space invasion, Overall survival, Stage IIIC disease

Gynecol Obstet Reprod Med 2021;27(1):70-76

1 Department of Gynecologic Oncology, Zekai Tahir Burak Women's Health Training and Research Hospital, Faculty of Medicine, University of Health Sciences, Ankara, Turkey

2 Department of Gynecologic Oncology, Etlik Zubeyde Hanim Women's Health Training and Research Hospital, Faculty of Medicine, University of Health Sciences, Ankara, Turkey

3 Department of Gynecologic Oncology, Kanuni Sultan Suleyman Teaching and Research Hospital, Faculty of Medicine, University of Health Sciences, Istanbul, Turkey

4Department of Obstetrics and Gynecology, Anadolu Medical Center, Kocaeli, Turkey

5 Division of Gynecologic Oncology, Department of Obstetrics and Gynecology, Faculty of Medicine, Baskent University, Ankara, Turkey

Address of Correspondence: Zeliha Firat Cuylan

Department of Gynecologic Oncology,

Zekai Tahir Burak Women's Health Training and Research Hospital, Faculty of Medicine,

University of Health Sciences, Ankara, Turkey zelihafiratcuylan@gmail.com

Submitted for Publication: $\quad$ 20.02.2019

Revised for Publication: 22.03.2019

Accepted for Publication: $\quad 12.04 .2019$

\section{Introduction}

Lymph node (LN) metastasis is one of the most important prognostic factors for endometrial cancer (EC) (1). In 2009, the International Federation of Gynecology and Obstetrics (FIGO) classified stage IIIC EC as substages IIIC1 and IIIC2

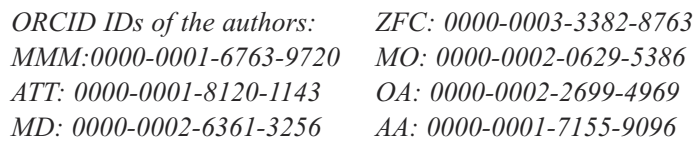

\begin{tabular}{|c|c|}
\hline Quick Response Code: & Access this article online \\
\cline { 2 - 2 } & Website: www.gorm.com.tr \\
\cline { 2 - 2 } & e- mail: info@gorm.com.tr \\
\cline { 2 - 3 } & DOI:10.201613/GORM.2019.925 \\
\hline
\end{tabular}

How to cite this article: Firat Cuylan Z. Meydanli MM. Oz M. Turan AT. Akbayir O. Dede M. Ayhan A. Comparison of Survival Outcomes of Lymphovascular Space Invasion-Negative and Lymphovascular Space Invasion - Positive Women with Stage IIIC Endometrioid Endometrial Cancer: A Case - Control Study. Gynecol Obstet Reprod Med. 2021;27(1):72-78 
according to pelvic and para-aortic LN involvement, respectively (2). Prognostic factors such as cervical stromal involvement, depth of myometrial invasion, vaginal involvement, uterine serosal involvement and adnexal metastasis do not alter FIGO stage when the patient has nodal metastasis (2). Although lymphovascular space invasion (LVSI) is not included in the FIGO staging classification for EC, several studies revealed LVSI as a prognostic factor for nodal metastasis and poor survival (3-5). However, studies on the effect of LVSI on survival outcomes in FIGO stage IIIC EC have conflicting results. Most of the studies have included non-endometrioid EC and carcinosarcomas along with the endometrioid EC patients, and they show heterogeneity in terms of adjuvant therapy $(4,6-8)$.

There is solid evidence in the literature about the prognostic significance of LVSI on survival and recurrence patterns in early stage EC (9-11). However, there are limited data about the prognostic significance of LVSI in advanced stage disease because of the heterogeneity of patients in advanced stages particularly in regards to adjuvant therapy, which varies across the institutions $(4,12,13)$. In this case-control study, we aimed to investigate the effect of LVSI on survival and recurrence patterns in patients with stage IIIC endometrioid type EC diagnosed by complete surgical staging including systematic pelvic and paraaortic lymphadenectomy.

\section{Material and Method}

Between November 1998 and November 2016, a multicenter retrospective analysis of patients with surgically staged EC at five gynecologic oncology institutions from Turkey was carried out, following the approval of the Local Institutional Review Boards (Approval number: 28/12/2017-6). All patients provided an informed consent for the surgical procedure and allowed the use of their medical information for research purposes at the time of admission and study was conducted in accordance with the Declaration of Helsinki.

We performed a retrospective database search from our prospectively maintained electronic database to find out patients diagnosed as stage IIIC Endometrioid type EC with LVSI negative. The case group consisted of 41 patients who had FIGO stage IIIC LVSI-negative endometrioid type EC according to the final pathology reports. For each patient with LVSI-negative endometrioid type EC, two women with FIGO stage IIIC LVSI-positive endometrioid type EC were selected from the same database for comparison. Cases and controls were matched in terms of age at diagnosis $(+/-10$ years $)$, grade of tumor (grade 1,2, or 3), sub-stage (IIIC1, IIIC2), adjuvant treatment and depth of myometrial invasion $(<1 / 2 \mathrm{vs} . \geq 1 / 2)$. Patients with non-endometrioid or mixed histologic types, women with inadequate lymphadenectomy, those with incomplete medical records and women with synchronous malignancies were excluded from the study. Data regarding patient medical histories, surgical staging and tumor pathology were gathered from the databases of the five institutions.
All patients underwent surgical staging with total hysterectomy \pm bilateral salpingo-oophorectomy, pelvic and paraaortic lymphadenectomy, infracolic omentectomy and peritoneal washings by gynecologic oncologists. Lymph node dissection was performed as previously described (14). Adequate pelvic and para-aortic lymphadenectomy have been defined as removal of at least 10 pelvic lymph nodes (LNs) and at least 5 para-aortic LNs (15-17).

Gynecologic pathologists examined and interpreted all surgical specimens but pathology reports were not reviewed centrally. On hematoxylin-eosin (H\&E) stained sections, the presence of LVSI was considered positive when tumor emboli were noted within a space clearly defined by endothelial cells (5). Architectural grading was defined by standard FIGO criteria (18). The histologic classification was performed according to the World Health Organization (WHO) classification (19). All tumors were staged using the 2009 FIGO staging system (2). In patients treated before 2009, the stage was determined retrospectively on the basis of surgical and pathologic assessment.

The treatment policies were decided by the attending physician or by the multidisciplinary tumor board at each participating institution. Adjuvant therapy was administered to all patients. Patients usually received chemoradiation with or without para-aortic irradiation and with or without brachytherapy.

Postoperative cancer surveillance included follow-up visits quarterly for the first 2 years, and biannually thereafter. The visits included a gynecologic medical history and a gynecologic examination that was further supplemented with biopsies in case of suspicious findings and imaging studies in case of suspicion of distant metastases. All women included in the study were followed until death or to the end of the study period (31 $1^{\text {st }}$ December 2016). The survival status of the patients was determined as alive or dead at the time of the last followup. For all study subjects with a recorded death, this was confirmed by performing a social security death index search.

Recurrence was defined as documentation of metastasis with physical examination and/or imaging techniques after a progression free survival (PFS) $\geq 3$ months. PFS was defined as the time from surgery to the first identification of recurrence or progression, or death from any cause, whichever occurred first, or the date of the last contact for patients remaining alive without recurrent disease. Overall survival (OS) was calculated as the time period between initial surgery to the date of death or the last contact. Surviving patients were censored at their last known follow-up.

Survival analysis was based on the Kaplan-Meier method, and the results were compared using a log-rank test. The chisquare test and Student's t-test for unpaired data were used for statistical analysis. Cox regression analysis was used to determine factors affecting survival, presented as hazard ratios (HRs) and 95\% confidence interval (CI), unadjusted or ad- 
justed for all factors. All variables with a $p$ value $<0.05$ in the univariate analysis were included in the multivariate analysis. All statistical analyses were performed with the SPSS software version 23.0 (SPSS, Inc., Chicago, IL). A p value $<0.05$ was considered to indicate statistical significance.

\section{Results}

Forty-one patients, who were identified with a postoperative pathology proven the diagnosis of stage IIIC LVSI-negative endometrioid type EC at participating institutions, were eligible for the study group. In order to match for comparison, 82 patients with LVSI-positive stage IIIC EC were also se- lected from the same institutions' databases using a dependent random sampling method.

Demographic and clinicopathologic characteristics of the study population are shown in table I. Age, menopausal status, primary tumor diameter, positivity of peritoneal cytology, adnexal and cervical involvement were similar between the cases and controls. The median age of patients in the LVSI negative group was 58 years (range; $40-75$ years); whereas it was 58.5 years (range; 30-82 years) in the LVSI-positive group $(p=0.77)$.

Median follow-up time for the LVSI-negative group was

Table I: Demographic and clinicopathologic characteristics of the study population $(n=123)$

\begin{tabular}{|c|c|c|c|}
\hline Characteristics & $\begin{array}{l}\text { LVSI-negative group } \\
\text { Values, n (\%) }\end{array}$ & $\begin{array}{l}\text { LVSI-positive group } \\
\text { Values, n (\%) }\end{array}$ & $p$ \\
\hline Age, y (median, range) & $58(40-75)$ & $58,5(30-85)$ & 0.77 \\
\hline Menopausal status & & & 0.09 \\
\hline Premenopausal & $4(9.8 \%)$ & $18(22 \%)$ & \\
\hline Postmenopausal & $37(90.2 \%)$ & $64(88 \%)$ & \\
\hline Tumor size (mean, SD, cm) & $4.2 \pm 2$ & $4.8 \pm 1.8$ & 0.09 \\
\hline \multicolumn{4}{|l|}{ Stage } \\
\hline IIIC1 & $24(58.5 \%)$ & $48(58.5 \%)$ & 1 \\
\hline IIIC2 & $17(41.5 \%)$ & $34(41.5 \%)$ & \\
\hline \multicolumn{4}{|l|}{ Grade } \\
\hline 1 & $12(29.2 \%)$ & $24(29.2 \%)$ & 1 \\
\hline 2 & $17(41.5 \%)$ & $34(41.5 \%)$ & \\
\hline 3 & $12(29.2 \%)$ & $24(29.2 \%)$ & \\
\hline \multicolumn{4}{|l|}{ MMI } \\
\hline$<50 \%$ & $12(29.3 \%)$ & $24(29.3 \%)$ & 1 \\
\hline$\geq 50 \%$ & $29(70.7 \%)$ & $58(70.7 \%)$ & \\
\hline \multicolumn{4}{|l|}{$\begin{array}{l}\text { Number of LN removed } \\
\text { (median, range) }\end{array}$} \\
\hline Total LN & $45(15-94)$ & $44.5(20-166)$ & 0.71 \\
\hline Pelvic LN & $34(10-64)$ & $32(10-105)$ & 0.91 \\
\hline Para-aortic LN & $15(5-36)$ & $14(5-79)$ & 0.33 \\
\hline \multicolumn{4}{|c|}{ Number of metastatic LN (median, range) } \\
\hline Total LN & $2(1-13)$ & $2(1-50)$ & 0.65 \\
\hline Pelvic LN & $2(0-13)$ & $2(0-37)$ & 0.94 \\
\hline Para-aortic LN & $0(0-9)$ & $0(0-13)$ & 0.80 \\
\hline \multicolumn{4}{|l|}{ Cervical involvement } \\
\hline Present & $10(24.4 \%)$ & $34(41.5 \%)$ & 0.06 \\
\hline Absent & $31(75.6 \%)$ & $48(58.5 \%)$ & \\
\hline \multicolumn{4}{|l|}{ Adnexal metastasis } \\
\hline Present & $5(12.2 \%)$ & $13(15.8 \%)$ & 0.58 \\
\hline Absent & $36(87.8 \%)$ & $69(84.2 \%)$ & \\
\hline \multicolumn{4}{|l|}{ Peritoneal cytology } \\
\hline Positive & $4(9.8 \%)$ & $17(20.7 \%)$ & 0.58 \\
\hline Negative & $37(90.2 \%)$ & $65(79.3 \%)$ & \\
\hline \multicolumn{4}{|l|}{ Adjuvant treatment } \\
\hline Chemotherapy & $8(19.5 \%)$ & $16(19.5 \%)$ & 1 \\
\hline Radiotherapy & $10(24.4 \%)$ & $20(24.4 \%)$ & \\
\hline Chemoradiotherapy & $23(56.1 \%)$ & $46(56.1 \%)$ & \\
\hline Number of Recurrences & $7 / 41(17.0 \%)$ & $20 / 81(24.4)$ & 0.29 \\
\hline Follow up, months (median, range) & $46(5-228)$ & $35(8-174)$ & 0.12 \\
\hline
\end{tabular}


46 months (range; 5-228) and it was 35 months (range; 8-174) for the LVSI-positive group ( $p=0.12$ ). The 5 -year PFS rates were comparable between the LVSI-negative and LVSI-positive groups ( $74.7 \%$ vs $67.8 \%$ respectively; $p=0.15$ ) (Figure 1$)$. The 5-year OS rates were $94.4 \%$ in the LVSI-negative group and $77.2 \%$ in the LVSI-positive group ( $p=0.058$ ) (Figure 2 ). During the follow-up period, 19 patients died (3/41 and 16/82 patients in the LVSI negative and positive groups, respectively). There was a trend towards statistical significance in terms of 5-year OS rates between the LVSI-negative and LVSI-positive groups.

Of 123 patients in the entire cohort, 27 (21.9\%) had recurrence during the study period. Recurrence rates were $17 \%$ (7/41) and 24.4\% (20/82) in LVSI-negative and LVSI-positive

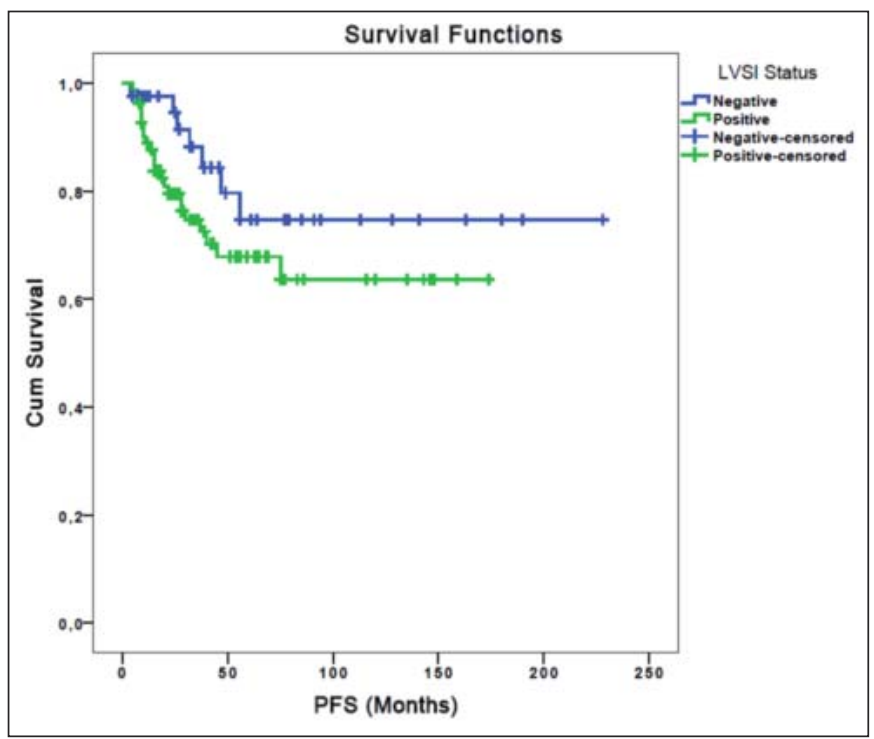

Figure 1: Comparison of progression-free survival between LVSI-negative and LVSI-positive women with stage IIIC endometrioid endometrial cancer

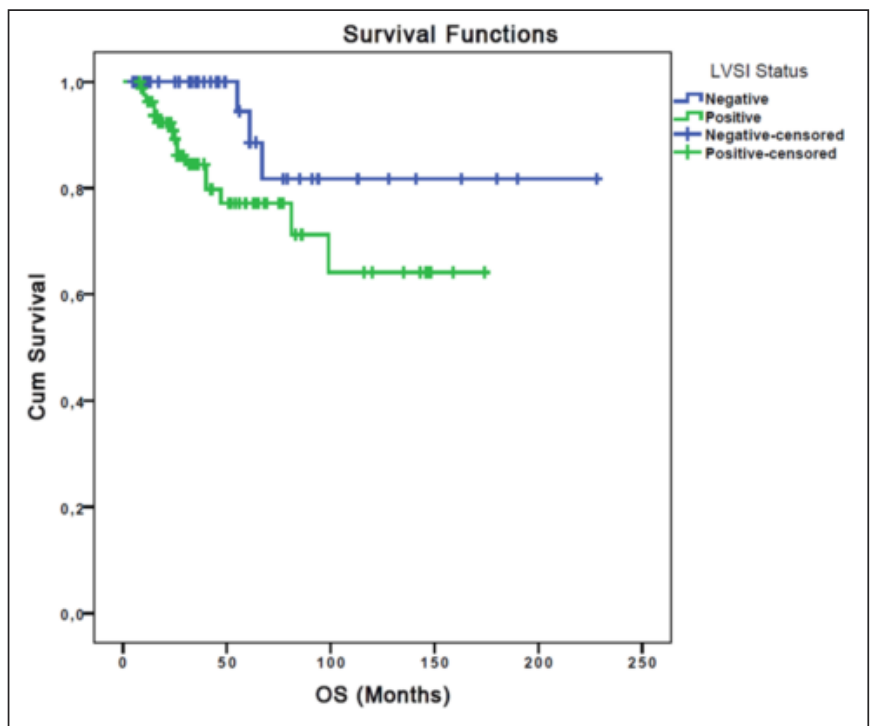

Figure 2: Comparison of overall survival between LVSI-negative and LVSI-positive women with stage IIIC endometrioid endometrial cancer groups, respectively $(p=0.48)$. There were $2(4.9 \%)$ cases of loco-regional and $5(12.2 \%)$ cases of distant recurrences and no retroperitoneal recurrences was observed in the LVSI-negative group. However, in the LVSI-positive group there were $6(7.3 \%)$ loco-regional, $9(10.9 \%)$ distant and $5(6.1 \%)$ retroperitoneal failures. There were 5 cases of retroperitoneal recurrences in the LVSI-positive group and no retroperitoneal recurrences in the LVSI-negative group.

For the entire cohort, univariate analysis revealed only age ( $\geq 58$ vs $<58$ years) $(p=0.002)$ as a significant factor for decreased PFS. In multivariate analysis, advanced age $(\geq 58$ years) (HR 3.52; 95\% CI: 1.51-8.21, $p=0.004$ ) remained as the only independent risk factor for decreased PFS.

For the entire cohort, univariate analysis revealed age $(\geq 58$ vs $<58$ years) $(p=0.004)$, grade (grade 3 vs $1-2)(p=0.003)$ and cervical involvement $(p=0.02)$ as significant factors for decreased OS. Multivariate analysis demonstrated advanced age ( $\geq 58$ years) (HR 3.73; 95\% CI: 1.19-11.69, $p=0.02$ ), grade 3 tumor (HR 2.57; 95\% CI: 1.002-6.62, $p=0.04$ ) and presence of cervical involvement (HR 2.62; 95\% CI: 1.03-6.67, $p=0.04$ ) as independent predictors of decreased OS (Table II). Additionally, there was a trend towards statistical significance between the presence of LVSI and OS in regression analysis (HR 3.10; 95\% CI 0.90-10.67, $p=0.05$ ) (Table II).

\section{Discussion}

In this multicenter case-control study, we found that there was a trend towards statistical significance in terms of OS rates between LVSI-negative and LVSI-positive patients in stage IIIC endometrioid EC (94.4\% vs $77.2 \%, p=0.058)$. A total of 27 (21.9\%) patients relapsed during the follow-up period. Recurrence rates were $17.0 \%$ (7/41) and 24.4\% (20/82) in the LVSI-negative and LVSI-positive groups, respectively ( $p=0.48$ ). There were 5 cases of retroperitoneal recurrences in the LVSI-positive group whereas no retroperitoneal recurrence was observed among LVSI-negative patients.

Patients in FIGO stage IIIC are heterogeneous in regards to the level of nodal involvement as well as extra-nodal involvement such as cervical stromal involvement, adnexal metastasis, uterine serosal involvement, vaginal involvement and adjuvant therapy. The 5-year OS rate and 5-year disease free survival (DFS) rate were reported with a wide range between $60 \%$ to $84 \%$ and $34 \%$ to $81 \%$, respectively in the literature (20-23). In terms of OS rate comparing to the previous studies, the 5-year OS rate was found to be better in the LVSInegative patients $(94.4 \%)$ in the current study. The worse survivals of patients in various previous studies might be explained by the inclusion of non-endometrioid tumors in these studies (20-23).

Narayan et al. (24) investigated the prognostic significance of LVSI in a retrospective cohort of intermediate and high-risk 
Table II: Univariate and multivariate analyses for overall survival in the entire cohort (Stage IIIC endometrioid adenocarcinoma LVSI negative ( $n=41)$ and positive $(n=82)$ groups

\begin{tabular}{|c|c|c|c|c|c|}
\hline & \multirow{2}{*}{$\begin{array}{c}\text { Univariate analysis OSa } \\
\quad n \text { of events }(\%)\end{array}$} & \multicolumn{4}{|c|}{ Multivariate Analysis of OSa } \\
\hline & & $p$ & HR & $\mathrm{Cl} 95 \%$ & $p$ \\
\hline \multicolumn{6}{|l|}{ Menopausal Status } \\
\hline Premenopausal & $1 / 22(95.2 \%)$ & 0.20 & & & \\
\hline Postmenopausal & $14 / 101(79.8 \%)$ & & & & \\
\hline \multicolumn{6}{|l|}{ Tumor Diameter, cm } \\
\hline$\leq 4$ & $6 / 58(86.1 \%)$ & 0.08 & & & \\
\hline$>4$ & $9 / 65(79.9 \%)$ & & & & \\
\hline \multicolumn{6}{|l|}{ LVSI } \\
\hline Negative & $1 / 41(94.4 \%)$ & 0.05 & & & \\
\hline Positive & $14 / 82(77.2 \%)$ & & & & \\
\hline \multicolumn{6}{|l|}{ MMI } \\
\hline$<50 \%$ & $1 / 36(97.0 \%)$ & 0.24 & & & \\
\hline$\geq 50 \%$ & $14 / 87(75.9 \%)$ & & & & \\
\hline \multicolumn{6}{|l|}{ Stage } \\
\hline IIIC1 & $10 / 72(82.8 \%)$ & 0.39 & & & \\
\hline IIIC2 & $5 / 51(82.4 \%)$ & & & & \\
\hline \multicolumn{6}{|l|}{ Adnexal Metastasis } \\
\hline Present & $2 / 18(86.5 \%)$ & 0.26 & & & \\
\hline Absent & $13 / 105(82.6 \%)$ & & & & \\
\hline \multicolumn{6}{|l|}{ Peritoneal Cytology } \\
\hline Positive & $5 / 21(85.7 \%)$ & 0.18 & & & \\
\hline Negative & $10 / 102(85.7 \%)$ & & & & \\
\hline \multicolumn{6}{|l|}{ Number of Metastatic LN } \\
\hline 1 & $5 / 38(84.2 \%)$ & 0.78 & & & \\
\hline$\geq 2$ & $10 / 85(82.7 \%)$ & & & & \\
\hline \multicolumn{6}{|l|}{ Age, y } \\
\hline$<58$ & $4 / 59(83.9 \%)$ & 0.004 & & & \\
\hline$\geq 58$ & $13 / 64(70.9 \%)$ & & 3.73 & $1.19-11.6$ & 0.02 \\
\hline \multicolumn{6}{|l|}{ Grade } \\
\hline $1-2$ & $7 / 87(89.0 \%)$ & 0.003 & & & \\
\hline 3 & $8 / 36(65.3 \%)$ & & 2.57 & $1.002-6.62$ & 0.04 \\
\hline \multicolumn{6}{|l|}{ Cervical Involvement } \\
\hline Present & $8 / 44(75.4 \%)$ & 0.02 & 2.62 & $1.03-6.67$ & 0.04 \\
\hline Absent & $7 / 79(86.7 \%)$ & & & & \\
\hline
\end{tabular}

a: 5-year overall survival rate, Cl: Confidence interval, HR: Hazard ratio, LVSI: Lymphovascular space invasion, MMI: Myometrial Invasion, n: Number, LN: Lymph node, OS: Overall survival, y: Year

EC patients. Sixty-five of the patients had nodal involvement with endometrioid histology; 56 of them had LVSI positive tumors, while only the remaining 9 patients had LVSI negative tumors. The recurrence rate was $35.7 \%$ in LVSI- positive and $11.1 \%$ in LVSI-negative patients among all node positive patients. The corresponding figures in the current study were $24.4 \%(20 / 82)$ in the LVSI-negative group and $17.0 \%(7 / 41)$ in the LVSI-positive group. However, Jorge et al. (25) found that LVSI remained associated with survival in node negative patients (HR 2.06, 95\% CI 1.65-2.58), but it was not associated with survival in women who already had nodal metastasis (HR 1.19; 95\% CI, 0.70-2.00). In our cohort, although there was a trend towards statistical significance between the presence of LVSI and OS (HR 3.10; 95\% CI 0.90-10.67, $p=0.05)$, this finding was not statistically significant.

Polterauer et al. (8) evaluated clinicopathologic parame- ters of 216 patients with stage IIIC endometrioid and non-endometrioid EC. In their cohort, LVSI was evident in $69.9 \%$ of the patients. Univariate analysis showed that LVSI had negative effect on survival, however multivariate analysis did not reveal this effect. In a retrospective study evaluating prognostic factors in FIGO stage IIIC endometrial cancer by Hoesktra et al. (13), age, myometrial invasion and non-endometrioid histology were associated with survival, but LVSI was not. However, in this study, $51.8 \%$ of patients had non-endometrioid histology, whereas LVSI was positive in $67.9 \%$ of the patients. In the current study, advanced age ( $\geq 58$ years) (HR 3.73; 95\% CI, 1.19-11.69, $p=0.02$ ), grade 3 tumors (HR 2.57; $95 \% \mathrm{CI}, 1.002-6.62, p=0.04)$ and presence of cervical involvement (HR 2.62; 95\% CI: 1.03-6.67, $p=0.04$ ) were independent predictors of decreased OS in the node positive women with endometrioid EC. 
Forty-six patients with stage IIIC endometrioid EC were evaluated by Mariani et al. (26) in 2002. Of the 46 patients, 22 had LNs as the only site of extra-uterine disease and 24 had also positive peritoneal cytology, uterine serosal, adnexal or vaginal involvement in addition to nodal involvement. Patients with nodal involvement only, had higher rates of LVSI positivity compared to the patients with both nodal and extra-nodal involvement ( $83 \%$ and 59\% respectively). In their cohort, LN recurrence rates were higher compared to our patients [14/46 (30\%) and 5/123(5\%), respectively]. All the patients with LN recurrences had positive LVSI status in our study but Mariani et al. (26) presented no data about LVSI status of the patients with LN recurrences in their study. They showed that LVSI-positive patients had worse cause specific survival (CSS) and recurrence free survival when compared to LVSI-negative patients ( $52 \%$ vs $75 \%, p=0.04 ; 41 \%$ vs $75 \%$, $p=0.03$ ) in their study (26). In the current study, we found that LVSI- positive patients had lower 5-year OS rates when compared to LVSI-negative patients $(94.4 \%$ vs $77.2 \%, p=0.058)$, however this finding was not statistically significant. This might be due to the limited number of patients in both groups.

In a multicenter study in 2015, 407 patients with stage IIIC endometrioid-type EC were analyzed. The predictors of DFS were parametrial invasion (HR 3.49; 95\% CI 1.83-6.64, $p<0.001$ ), higher grade (HR 2.78; 95\% CI 1.31-5.89, $p=0.008$ ) and $>3$ positive pelvic nodes (HR 1.84; 95\% CI 1.11-3.05, $p=0.019$ ). Predictors of OS were parametrial invasion (HR 4.43; 95\% CI, 2.01-9.76, $p<0.001$ ), older age (HR 3.82; 95\% CI, 1.93-7.56; $p<0.001)$ and $>3$ positive pelvic nodes (HR 2.62; 95\% CI 1.35-5.06; $p=0.004)$ but LVSI had no effect on 5 year-DFS $(p=0.38)$ and 5 year-OS $(p=0.55)$. (12). In the current study, for the entire cohort, advanced age ( $\geq 58$ years) (HR 3.73 ; 95\% CI, 1.19-11.69, $p=0.02$ ), grade 3 tumors (HR 2.57; $95 \% \mathrm{CI}, 1.002-6.62, p=0.04)$ and presence of cervical involvement (HR 2.62; 95\% CI, 1.03-6.67, $p=0.04$ ) were found as independent predictors of decreased OS.

The major limitation of our study is its retrospective design. Lack of central pathology review might be the second drawback of the current study. Some previous studies provided central pathology confirmation of the specimens, but the retrospective multicenter design of our study made it impossible for us to obtain paraffin embedded specimens of all patients. Although the present study is multicentric, the number of patients in the case group was relatively low due to low incidence of LVSI-negative stage IIIC endometrioid EC patients.

The major strength of the current study is its design. We designed it as a case-control study in order to eliminate confounding factors for survival by matching the age, stage, grade, myometrial invasion and adjuvant therapy and addressing the LVSI status as the main variable. To the best of our knowledge, the current study is the first case-control study evaluating the effect of LVSI on survival in stage IIIC endometrioid EC.
In conclusion, in the current study, we found that patients with stage IIIC endometrioid EC with positive LVSI status had worse survival, but this finding was not statistically significant. We also found nodal recurrence rate was higher in patients with LVSI. In the light of our findings, LVSI status seems to be of borderline significance for OS in stage IIIC endometrioid EC and impacts recurrence patterns as retroperitoneal recurrences are strongly related to positive LVSI status.

Acknowledgements: None.

Funding: None

Conflict of Interest: The authors declare no conflict of interest in the preparation and publishing the study.

Authors' contributions: ZFC: Conceptualization, data curation, investigation, methodology, software, validation, writing, review, editing. MMM: Conceptualization, data curation, formal analysis, investigation, methodology, software, supervision, validation, writing, review, editing. MO: Data curation, formal analysis, investigation, methodology, software, writing review, editing. ATT: Conceptualization, formal analysis, methodology, supervision, validation, writing, review, editing. OA: Data curation, formal analysis, methodology, supervision, visualization. MD: Conceptualization, methodology, supervision, validation, visualization, writing, review, editing AA: Conceptualization, formal analysis, methodology, supervision, visualization, writing, review, editing.

\section{References}

1. Creasman WT. Morrow CP. Bundy BN. Homesley HD. Graham JE. Heller PB. Surgical pathologic spread patterns of endometrial cancer. A Gynecologic Oncology Group Study. Cancer. 1987;60(8 Suppl):2035-41. doi: 10.1002/1097-0142(19901015)60:8+<2035::aid-cncr 2820601515>3.0.co;2-8.

2. Pecorelli S. Revised FIGO staging for carcinoma of the vulva, cervix, and endometrium. Int J Gynaecol Obstet. 2009;105(2):103-4. doi: 10.1016/j.ijgo.2009.02.012.

3. Hahn HS. Lee IH. Kim TJ. Lee KH. Shim JU. Kim JW. et al. Lymphovascular space invasion is highly associated with lymph node metastasis and recurrence in endometrial cancer. Aust N Z J Obstet Gynaecol. 2013;53(3):293-7. doi: 10.1111/ajo.12089.

4. Watari H. Todo Y. Takeda M. Ebina Y. Yamamoto R. Sakuragi N. Lymph-vascular space invasion and number of positive para-aortic node groups predict survival in node-positive patients with endometrial cancer. Gynecol Oncol. 2005;96(3):651-7. doi: 10.1016/j.ygyno.2004. 11.026 .

5. Cheewakriangkrai C. Panggid K. Siriaungkul S. Khunamornpong S. Suprasert P. Srisomboon J. Lymphovascular space invasion as a prognostic determinant in uterine cancer. Asian Pac J Cancer Prev. 2007;8(3):363-6.

6. Turan T. Ureyen I. Duzguner I. Ozkaya E. Tasci T. 
Karalok A. et al. Analysis of patients with stage IIIC endometrial cancer. Int J Gynecol Cancer. 2014;24(6):103341. doi: 10.1097/IGC.0000000000000154.

7. Toptas T. Simsek T. Stage IIIC endometrial cancer: the need for novel subgrouping according to the ratio of metastatic lymph nodes. Arch Gynecol Obstet. 2015;291 (2):391-8. doi: 10.1007/s00404-014-3409-z.

8. Polterauer S. Khalil S. Zivanovic O. Abu-Rustum NR. Hofstetter G. Concin N. et al. Prognostic value of lymph node ratio and clinicopathologic parameters in patients diagnosed with stage IIIC endometrial cancer. Obstet Gynecol. 2012;119(6):1210-8. doi: 10.1097/AOG. 0b0 $13 \mathrm{e} 318255060 \mathrm{c}$.

9. dos Reis R. Burzawa JK. Tsunoda AT. Hosaka M. Frumovitz M. Westin SN. et al. Lymphovascular Space Invasion Portends Poor Prognosis in Low-Risk Endometrial Cancer. Int J Gynecol Cancer. 2015;25(7): 1292-9. doi: 10.1097/IGC.0000000000000490.

10. Briët JM. Hollema H. Reesink N. Aalders JG, Mourits MJ. ten Hoor KA. et al. Lymphvascular space involvement: an independent prognostic factor in endometrial cancer. Gynecol Oncol. 2005;96(3):799-804. doi: 10. 1016/j.ygyno.2004.11.033.

11. Gemer O. Arie AB. Levy T. Gdalevich M. Lorian M. Barak F. et al. Lymphvascular space involvement compromises the survival of patients with stage I endometrial cancer: results of a multicenter study. Eur J Surg Oncol. 2007;33(5):644-7. doi: 10.1016/j.ejso.2007.01.009.

12. Yoon MS. Park W. Huh SJ. Kim HJ. Kim YS. Kim YB. et al. A multicenter analysis of adjuvant therapy after surgery for stage IIIC endometrial adenocarcinoma: A Korean Radiation Oncology Group study (KROG 13-17). Gynecol Oncol. 2015;138(3):519-25. doi: 10.1016/ j.ygyno.2015.06.030.

13. Hoekstra AV. Kim RJ. Small W Jr. Rademaker AW. Helenowski IB. Singh DK. et al. FIGO stage IIIC endometrial carcinoma: prognostic factors and outcomes. Gynecol Oncol. 2009;114(2):273-8. doi: 10.1016/ j.ygyno.2009.04.013.

14. Ayhan A. Topfedaisi Ozkan N. Oz M. Kimyon Comert G. Firat Cuylan Z. Coban G. et al. Impact of lymph node ratio on survival in stage IIIC endometrioid endometrial cancer: a Turkish Gynecologic Oncology Group study. J Gynecol Oncol. 2018;29(4):e48. doi: 10.3802/jgo.2018.29.e48.

15. Mariani A. Webb MJ. Keeney GL. Haddock MG. Calori G. Podratz KC. Low-risk corpus cancer: is lymphadenectomy or radiotherapy necessary? Am J Obstet Gynecol. 2000;182(6):1506-19. doi: 10.1067/mob.2000.107335.

16. Keys HM. Roberts JA. Brunetto VL. Zaino RJ. Spirtos NM. Bloss JD. et al. A phase III trial of surgery with or without adjunctive external pelvic radiation therapy in intermediate risk endometrial adenocarcinoma: a Gynecologic Oncology Group study. Gynecol Oncol. 2004;92(3):744-51. doi: 10.1016/j.ygyno.2003.11.048.
17. Nomura H. Aoki D. Suzuki N. Susumu N. Suzuki A. Tamada Y. et al. Analysis of clinicopathologic factors predicting para-aortic lymph node metastasis in endometrial cancer. Int J Gynecol Cancer. 2006;16(2):799-804. doi: 10.1111/j.1525-1438.2006.00529.x.

18. Werner HM. Trovik J. Marcickiewicz J. Tingulstad S. Staff AC. Amant F. et al. Revision of FIGO surgical staging in 2009 for endometrial cancer validates to improve risk stratification. Gynecol Oncol. 2012;125(1):103-8. doi: 10.1016/j.ygyno.2011.11.008.

19. Creasman WT. Odicino F. Maisonneuve P. Quinn MA. Beller U. Benedet JL. et al. Carcinoma of the corpus uteri. FIGO 26th Annual Report on the Results of Treatment in Gynecological Cancer. Int J Gynaecol Obstet. 2006;95 Suppl 1:S105-43. doi: 10.1016/S0020-7292(06)60031-3.

20. Lee LJ. Viswanathan AN. Combined chemotherapy and radiation improves survival for node-positive endometrial cancer. Gynecol Oncol. 2012;127(1):32-7. doi: 10.1016/ j.ygyno.2012.06.026.

21. Klopp AH. Jhingran A. Ramondetta L. Lu K. Gershenson DM. Eifel PJ. Node-positive adenocarcinoma of the endometrium: outcome and patterns of recurrence with and without external beam irradiation. Gynecol Oncol. 2009;115(1):6-11. doi: 10.1016/j.ygyno.2009.06.035.

22. Mundt AJ. Murphy KT. Rotmensch J. Waggoner SE. Yamada SD. Connell PP. Surgery and postoperative radiation therapy in FIGO Stage IIIC endometrial carcinoma. Int J Radiat Oncol Biol Phys. 2001;50(5):1154-60. doi: 10.1016/s0360-3016(01)01590-5.

23. Nelson G. Randall M. Sutton G. Moore D. Hurteau J. Look K. FIGO stage IIIC endometrial carcinoma with metastases confined to pelvic lymph nodes: analysis of treatment outcomes, prognostic variables, and failure patterns following adjuvant radiation therapy. Gynecol Oncol. 1999;75(2):211-4. doi: 10.1006/gyno.1999.5569.

24. Narayan K. Khaw P. Bernshaw D. Mileshkin L. Kondalsamy-Chennakesavan S. Prognostic significance of lymphovascular space invasion and nodal involvement in intermediate- and high-risk endometrial cancer patients treated with curative intent using surgery and adjuvant radiotherapy. Int J Gynecol Cancer. 2012;22(2):260-6. doi: 10.1097/IGC.0b013e318230c264.

25. Jorge S. Hou JY. Tergas AI. Burke WM. Huang Y. Hu JC. et al. Magnitude of risk for nodal metastasis associated with lymphvascular space invasion for endometrial cancer. Gynecol Oncol. 2016;140(3):387-93. doi: 10.1016/j.ygyno.2016.01.002.

26. Mariani A. Webb MJ. Keeney GL. Haddock MG. Aletti G. Podratz KC. Stage IIIC endometrioid corpus cancer includes distinct subgroups. Gynecol Oncol. 2002;87(1): 112-7. doi: 10.1006/gyno.2002.6789. 